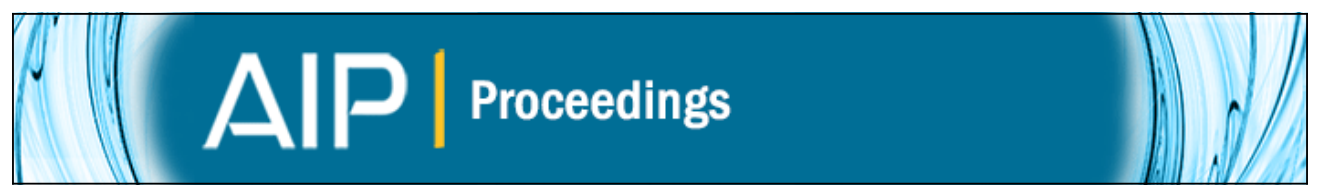

\title{
Asymmetric Time Evolution and Indistinguishable Events
}

P. W. Bryant

Citation: AIP Conference Proceedings 1307, 27 (2010); doi: 10.1063/1.3527422

View online: http://dx.doi.org/10.1063/1.3527422

View Table of Contents:

http://scitation.aip.org/content/aip/proceeding/aipcp/1307?ver=pdfcov

Published by the AIP Publishing

\section{Articles you may be interested in}

On Differential Expressions with $\delta$-Potential: Exceptional Case $d=2$ I

AIP Conf. Proc. 1307, 1 (2010); 10.1063/1.3527419

Some Boussinesq Equations with Saturation

AIP Conf. Proc. 1301, 553 (2010); 10.1063/1.3526657

An Explicitly Correlated Wavelet Method for the Electronic Schrödinger Equation AIP Conf. Proc. 1281, 933 (2010); 10.1063/1.3498647

Comparison of the Chebyshev Method and the Generalized Crank-Nicholson Method for time Propagation in Quantum Mechanics

AIP Conf. Proc. 1281, 667 (2010); 10.1063/1.3498565

Four-body reactions of light nuclei

AIP Conf. Proc. 1265, 88 (2010); 10.1063/1.3480268 


\title{
Asymmetric Time Evolution and Indistinguishable Events
}

\author{
P. W. Bryant \\ Center for Complex Quantum Systems, Department of Physics, University of Texas at Austin, \\ Austin, Texas 78712, USA
}

\begin{abstract}
With a time asymmetric theory, in which quantum mechanical time evolution is given by a semigroup of operators rather than by a group, the states of open systems are represented by density operators exhibiting a branching behavior. To treat the indistinguishably of the members of experimental ensembles, we hypothesize that environmental interference occurs during events that are themselves fundamentally indistinguishable.
\end{abstract}

Keywords: Time asymmetry, semigroup, coordinates, open quantum systems, indistinguishably PACS: 03.65.Ca,03.65.Yz,02.20.Uw

\section{ASYMMETRIC TIME EVOLUTION}

The theoretical image of any closed, quantum mechanical system is an operator algebra defined in a linear scalar-product space, $\Phi[1]$. The vectors $\phi_{n}$ span $\Phi$, and every linear combination of the $\phi_{n}$ can represent the state of the system. For simplicity, in this section we shall consider a single vector, $\phi$, that spans a one-dimensional subspace of $\Phi$. The ray spanned by $\phi$ represents a quantum system in a pure state.

Let us work in the Schrödinger picture and assume that the system's dynamics is given by the Schrödinger equation,

$$
i \hbar \frac{\partial}{\partial t} \phi=H \phi
$$

As a constraint on the solutions, one has historically chosen $\phi \in \mathscr{H}$, where $\mathscr{H}$ is the Hilbert space. With this choice, Stone and von Neumann showed [2, 3] that (1) integrates to

$$
\phi(t)=e^{-\frac{i H t}{\hbar}} \phi, \quad-\infty<t<\infty .
$$

The solution $\phi$ evolves in time via the one-parameter group of unitary operators

$$
U(t)=e^{-\frac{i H t}{\hbar}}, \quad-\infty<t<\infty .
$$

The group product is $U\left(t_{1}\right) U\left(t_{2}\right)=U\left(t_{1}+t_{2}\right)$, and the inverse exists:

$$
(U(t))^{-1}=U(-t) .
$$

Because of (3), the time-reversed version of $\phi(t)$, which is $\phi(-t)$, is also a solution of the Schrödinger equation. When $\phi \in \Phi=\mathscr{H}$, the dynamics is symmetric in time. 
Suppose that, instead of the group in (2), time evolution is given by a one-parameter semigroup of operators

$$
U(t)=e^{-\frac{i H t}{\hbar}}, \quad 0 \leq t<\infty .
$$

Note the lower bound on the time parameter, $t$. While the product is unchanged, being a semigroup means that the inverse no longer exists. In other words, the time-reversed state, $\phi(-t)$, is not a solution of the Schrödinger equation. Such a dynamics is asymmetric in time.

This asymmetric time evolution can also be mathematically derived. For a variety of reasons, mostly concerning scattering theory [4], one chooses another possible constraint for $\phi$ :

$$
\left.\langle E \mid \phi\rangle \equiv \phi(E) \in\left(\mathscr{S} \cap \mathscr{H}_{-}^{2}\right)\right|_{\mathbb{R}_{+}},
$$

where $\mathscr{S}$ is the Schwartz space, and $\mathscr{H}_{-}^{2}$ is the space of Hardy class functions analytic in the lower half plane. The energy wave functions, $\phi(E)$, are thus required to be "well-behaved" functions that can be analytically continued into $\mathbb{C}_{-}$. This constraint (5) defines the scalar-product space for state vectors, which we will call $\Phi_{-}$:

$$
\phi \in \Phi_{-} \subset \mathscr{H} \subset \Phi_{-}^{\times},
$$

where $\Phi_{-}^{\times}$is the dual space. This triplet of spaces constitutes a Rigged Hilbert Space. For details, see [5].

By the Paley-Wiener theorem [6], with the constraint (6), the dynamical equation (1) integrates to [7]

$$
\phi(t)=e^{-\frac{i H t}{\hbar}} \phi, \quad 0 \leq t<\infty .
$$

This is precisely the asymmetric time evolution described by the semigroup (4). Given that energy wave functions can be continued into $\mathbb{C}_{-}$(5), the semigroup time evolution may come as no surprise to readers familiar with the Lippmann-Schwinger equation from scattering theory or with Feynman propagators from field theory.

\section{PHYSICAL INTERPRETATION OF TIME PARAMETERS}

We will distinguish between the time parameter for the group and the time parameter for the semigroup by writing $\tilde{t}$ for the group's parameter (2), and writing $t$ for the semigroup parameter (4). Considering the ranges from which values of $t$ or $\tilde{t}$ are chosen,

$$
t \in[0, \infty) \quad \text { and } \quad \tilde{t} \in(-\infty, \infty),
$$

this distinction is necessary.

For non-relativistic quantum mechanics, because $-\infty<\tilde{t}<\infty$, one identifies $\tilde{t}$ with "Newton's absolute," or coordinate, time [8]. Coordinate time has also been called "external" time because it is not dynamically linked to any quantum system that might be in a state represented by $\phi(\tilde{t})[9]$.

Because $t \geq 0, t$ is more naturally identified with durations in time. In contrast with time coordinate values, which are physically meaningless, durations are physically 
significant, and the $t$ has been called "intrinsic" time because it is dynamically linked to a quantum system in the state $\phi(t)[9]$.

If $T_{\delta}$ is a time translation operator defined on coordinates, then it acts on $\tilde{t}$ as

$$
T_{\delta} \tilde{t} \rightarrow \tilde{t}+\delta
$$

Durations are invariant under time translation, so

$$
T_{\delta} t \rightarrow t
$$

We shall hereafter call the $\tilde{t}$ time coordinates, and we shall call the $t$ time parameters.

\section{OPEN SYSTEMS}

One represents the state of a closed, dynamical system with a density operator, $\rho(t)$, which is constructed from the state vectors spanning the scalar-product space, $\Phi_{-}$, for the system. For the pure state discussed above, $\rho(t)=|\phi(t)\rangle\langle\phi(t)|$. By construction, $\rho(t)$ can describe the state of the closed system itself and of nothing external to it.

A closed system is an idealization, and every experimental system of interest suffers interaction with an uncontrolled, quantum mechanical environment. By definition, state vectors for the closed system cannot describe external interference. To treat interactions with external systems, then, one needs the theoretical image of composite systems made of the system of interest together with the uncontrolled environment systems [10]. If $\left(\Phi_{-}\right)_{1}$ contains vectors representing the state of the system of interest, one requires

- a space $\left(\Phi_{-}\right)_{2}$ for the composite system associated with one type of interaction,

- a space $\left(\Phi_{-}\right)_{3}$ for the system associated with another interaction, etc.

We shall denote by $\rho_{i}$ the density operator constructed from state vectors spanning the space $\left(\Phi_{-}\right)_{i}$.

Quantum mechanics makes probabilistic predictions, so for every useful experiment one makes measurements on an experimental ensemble containing as many members as possible, all identically prepared [11]. At preparation, all members are in a state represented by, say, $\rho_{1}\left(t_{1}\right)$. Because experimental systems are open, eventually environmental interference affects some subset of the ensemble. One then represents the state of the affected ensemble members by $\rho_{2}\left(t_{2}\right)$. The unaffected ensemble members continue to be in a state represented by $\rho_{1}\left(t_{1}\right)$. After another instance of environmental interference, one requires $\rho_{3}\left(t_{3}\right)$ to represent the state of affected members. The entire ensemble, then, is represented by

$$
a_{1} \rho_{1}\left(t_{1}\right)+a_{2} \rho_{2}\left(t_{2}\right)+a_{3} \rho_{3}\left(t_{3}\right) .
$$

In (7), $a_{1}+a_{2}+a_{3}=1$. Note that, because the $t_{i}$ correspond to durations and are not related to any value of the time coordinate, $\tilde{t}$, in general $t_{i} \neq t_{j \neq i}$ for a given $\tilde{t}$. For details, see [10] or Table 1.

This "branching" behavior of the density operator occurs whenever any subset of the experimental ensemble members suffers the environmental interference inevitable for open systems. In general, the equation (7) is not well-defined because the $\rho_{i}$ will not be 


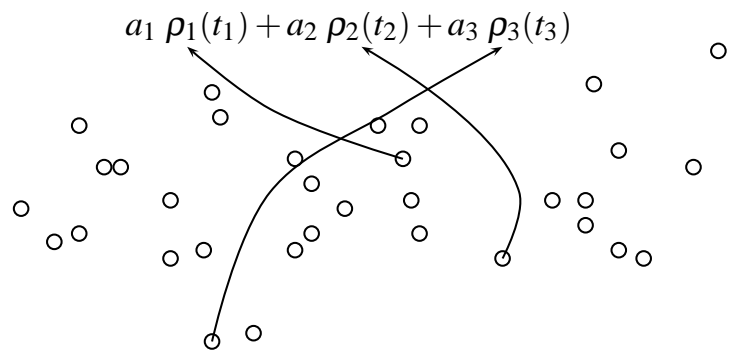

FIGURE 1. Schematically associating ensemble members with different branches. The circles represent members of the experimental ensemble.

defined in the same space. To treat it rigorously, to calculate Born probabilities, one must specify how an operator defined in $\left(\Phi_{-}\right)_{i}$ is extended or limited to the space $\left(\Phi_{-}\right)_{j \neq i}$.

An alternative treatment for open systems does exist, in which systems of interest are treated as subsystems of arbitrarily large environmental systems [12]. The useful result is often a decoherence master equation for the dynamics of the system of interest. The arbitrarily large environmental systems may include the clocks on the laboratory walls, and these clocks mark external, or coordinate time values, $\tilde{t}$. Both the system of interest and the environment evolve in time together, so consistency requires one to identify the time in the master equation with a time coordinate value, $\tilde{t}$. Because the $t$ in the semigroup (4) is a parameter, one cannot use this alternative treatment with time asymmetric theories.

\section{PARTITIONING AND PHYSICAL REQUIREMENTS}

The probability to find an observable, represented by the operator $\Lambda$, in the state $\rho$, is given by the Born probability

$$
\mathscr{P}_{\Lambda}\left(\rho\left(\tilde{t}-\tilde{t}_{0}\right)\right)=\operatorname{Tr}\left(\Lambda \rho\left(\tilde{t}-\tilde{t}_{0}\right)\right) .
$$

Measurements are performed in the coordinate time of the laboratory, so we have written (8) as a function of $\tilde{t}-\tilde{t}_{0}$, where $\tilde{t}_{0}$ is the time coordinate at which ensemble members are actively prepared.

To analyze an experiment, from a preparation procedure or from a measurement one infers the density operator, $\rho\left(\tilde{t}-\tilde{t}_{0}\right)$. With the branching density operators, the inferred density operator, $\rho\left(\tilde{t}-\tilde{t}_{0}\right)$, is compared to a calculated density operator with many branches that are functions of the parameters $t_{i}$, such as the one in (7). One then faces the interesting problem of determining which ensemble members are in states represented by which branch of the density operator. Consider the density operator with three branches from (7). Any model of an open system will depend on how the ensemble members are partitioned among the branches. A schematic of this is shown in Figure 1.

The $a_{i}$ in Figure 1 are determined by the partitioning scheme, and we can immediately make a strong, general requirement on models by noting that no model should ever de- 
TABLE 1. Relating timescales to time parameter values from (7). The pattern continues as more branches form.

\begin{tabular}{cccc}
\hline value of $\tilde{\mathbf{t}}-\tilde{\mathbf{t}}_{\mathbf{0}}$ & value of $\mathbf{t}_{\mathbf{1}}$ & value of $\mathbf{t}_{\mathbf{2}}$ & value of $\mathbf{t}_{\mathbf{3}}$ \\
\hline 0 & 0 & not defined & not defined \\
$1 \Delta \tilde{t}$ & $1 \Delta \tilde{t}$ & 0 & not defined \\
$2 \Delta \tilde{t}$ & $2 \Delta \tilde{t}$ & $1 \Delta \tilde{t}$ & 0 \\
$3 \Delta \tilde{t}$ & $3 \Delta \tilde{t}$ & $2 \Delta \tilde{t}$ & $1 \Delta \tilde{t}$
\end{tabular}

pend on how many measurements are made. Partitioning, therefore, must be independent of the size of the experimental ensemble.

Another requirement comes from the fact that the different members of experimental, quantum mechanical ensembles are fundamentally indistinguishable from each other. Upon the development of a third branch, for example,

$$
a_{1} \rho_{1}\left(t_{1}\right)+a_{2} \rho_{2}\left(t_{2}\right) \stackrel{\text { branching }}{\longrightarrow} a_{1} \rho_{1}\left(t_{1}\right)+a_{2} \rho_{2}\left(t_{2}\right)+a_{3} \rho_{3}\left(t_{3}\right),
$$

one cannot say which members represented by $\rho_{1}\left(t_{1}\right)$ on the left hand side have, due to environmental interaction, become representable by $\rho_{3}\left(t_{3}\right)$ on the right hand side, etc. To do so would require the labeling of individual ensemble members.

Based on physical considerations, then, we have the general requirements:

1. Models must be independent of ensemble size.

2. Indistinguishable ensemble members cannot be labeled.

The framework of branching density operators is flexible, but creating a reasonable model that meets both physical requirements can be quite complicated. To simplify models, we hypothesize the existence of indistinguishable events.

\section{INDISTINGUISHABLE EVENTS}

Tractable models for environmental interference with dynamical systems will often depend on a timescale for the interference, $\Delta \tilde{t} .^{1}$ This means that, for a given type of interference, on average the discontinuous branching process occurs at increments of $\Delta \tilde{t}: k \Delta \tilde{t}=1 \Delta \tilde{t}, 2 \Delta \tilde{t}, 3 \Delta \tilde{t} . . n \Delta \tilde{t}$. For a model with one type of interference and thus one timescale, time parameters, for example from (7), are related to durations in the lab according to Table 1 .

When an ensemble member suffers interference, its state is initially represented by $\rho_{i}\left(t_{i}=0\right)$. With Table 1 , knowing at which $k \Delta \tilde{t}$ a member suffers interference suffices to fix the appropriate branch, $\rho_{i}\left(t_{i}\right)$, allowing one to define the $a_{i}$. Both physical requirements above can be realistically met if we suppose that indistinguishable ensemble

\footnotetext{
${ }^{1}$ We write $\Delta \tilde{t}$, a duration in coordinate time, rather than $\Delta t$, a duration in parameter time, because interference time scales are inferred from measurement, which occurs in the coordinate time of the lab. Furthermore, parameters in the problem are all associated with the density matrix of the system of interest, and not with an uncontrolled environment.
} 
members are labeled by the particular $k$ corresponding to $k \Delta \tilde{t}$ at which they have suffered interference, and then that the individual $\Delta \tilde{t}$ 's are treated as indistinguishable. In effect, we hypothesize that the events in which systems suffer environmental interference are themselves indistinguishable.

To implement indistinguishable events, we parametrize the probability, $\beta$, that a given $\Delta \tilde{t}$ passed before an interference event. We use the binomial distribution to count the combinations with $k$ indistinguishable intervals, of length $\Delta \tilde{t}$, occurring before the duration $k \Delta \tilde{t}$, and thereby relate the label $k$ to the passage of time:

$$
\left(\begin{array}{l}
n \\
k
\end{array}\right) \beta^{k}(1-\beta)^{n-k} \Longleftrightarrow k \text { from } k \Delta \tilde{t} .
$$

Here the $n$ is related to the total number of intervals of length $\Delta \tilde{t}$ that have passed, and it is unrelated to the ensemble size. With this approach, we have thus satisfied the physical requirement that models be independent of the number of measurements.

A model with indistinguishable events was developed in [10], and it successfully resulted in quantitative agreement with previously puzzling experimental data. Encouraged by this, we hope to investigate further the measurable consequences of indistinguishable interference events.

\section{ACKNOWLEDGMENTS}

My sincerest thanks go to the organizers of the XXIX Workshop on Geometric Methods in Physics, in Białowieża, for their generous hospitality. In particular I wish to thank Dr. Odzijewicz, Dr. Kielanowski, Dr. Goliński, and all my friends at the Skansen.

\section{REFERENCES}

1. A. Bohm, Quantum Mechanics: Foundations and Applications, Springer-Verlag, 1993, 3rd edn.

2. M. H. Stone, Ann. Math. 33, 643 (1932).

3. J. von Neumann, Ann. Math. 33, 567 (1932).

4. A. Bohm, P. Bryant, and Y. Sato, J. Phys. A 41, 304019 (2008), ISSN 1751-8121.

5. A. Bohm, and M. Gadella, Dirac Kets, Gamow Vectors, and Gel'fand Triplets, Springer-Verlag, Germany, 1989.

6. R. Paley, and N. Wiener, Fourier transforms in the complex domain, American Mathematical Society, New York, 1934.

7. A. Bohm, P. Kielanowski, and S. Wickramasekara, Ann. Phys. 321, 2299-2317 (2006).

8. H. D. Zeh, "Time in Quantum Theory," in Compendium of Quantum Physics, edited by D. Greenberger, K. Hentschel, and F. Weinert, Springer, 2009, pp. 786-792.

9. P. Busch, Lect. Notes Phys. 734, 73-105 (2008).

10. P. W. Bryant, 0912.5363 (2010), URL http: / / arxiv . org/abs/0912.5363.

11. P. A. M. Dirac, The Principles of Quantum Mechanics, Oxford University Press, 1958, 4th edn.

12. H. Breuer, and F. Petruccione, The Theory of Open Quantum Systems, Oxford University Press, 2002. 